\title{
Effects of Oxide Layer Composition and Radial Compression on Nickel Release in Nitinol Stents
}

\author{
Stacey J. L. Sullivan ${ }^{1}$ Maureen L. Dreher ${ }^{1} \cdot$ Jiwen $_{\text {Zheng }}{ }^{1} \cdot$ Lynn Chen $^{1} \cdot$ \\ Daniel Madamba $^{2} \cdot$ Katie Miyashiro $^{2} \cdot$ Christine Trépanier $^{2} \cdot$ Srinidhi Nagaraja $^{1}$
}

Published online: 30 July 2015

(C) ASM International 2015

\begin{abstract}
There is a public health need to understand the effects of surface layer thickness and composition on corrosion in nickel-containing medical devices. To address this knowledge gap, five groups of Nitinol stents were manufactured by various processing methods that altered the titanium oxide layer. The following surfaces were created: $>3500 \mathrm{~nm}$ thick mixed thermal oxide (OT), $\sim 420 \mathrm{~nm}$ thick mixed thermal oxide (SP), $\sim 130 \mathrm{~nm}$ thick mixed thermal oxide (AF), $\sim 4 \mathrm{~nm}$ thick native oxide (MP), and an $\sim 4 \mathrm{~nm}$ thick passivated oxide (EP). Radially compressed and not compressed devices were evaluated for nickel $(\mathrm{Ni})$ ion release in a 60-day immersion test. The results indicated that OT stents released the most Ni, followed by stents in the SP and AF groups. For OT and SP stents, which exhibited the thickest oxide layers, radial compression significantly increased Ni release when compared to non-compressed stents. This result was not observed in AF, MP, SP stents indicating that the increased $\mathrm{Ni}$ release may be explained by cracking of the thicker oxide layers during crimping. Strong correlations were observed between oxide layer thickness and cumulative $\mathrm{Ni}$ release. These findings elucidate the importance of oxide
\end{abstract}

This article is an invited paper selected from presentations at the International Conference on Shape Memory and Superelastic Technologies 2014, held May 12-16, 2014, in Pacific Grove, California, and has been expanded from the original presentation.

Srinidhi Nagaraja

srinidhi.nagaraja@fda.hhs.gov

1 Center for Devices and Radiological Health, Office of Science and Engineering Laboratories, U.S. Food and Drug Administration, 10903 New Hampshire Avenue, Building 62, Room 2210, Silver Spring, MD 20993-0002, USA

2 Nitinol Devices and Components, Fremont, CA, USA layer thickness and composition on uniform corrosion of laser-cut Nitinol stents.

Keywords Shape memory stents - Biocompatibility · Nitinol $\cdot$ Corrosion $\cdot$ Nickel release $\cdot$ Crimp $\cdot$ Titanium oxide $\cdot$ Thermal oxidation

\section{Introduction}

Nitinol is a commonly used medical device material due to its unique pseudoelastic and shape memory properties. The ability of Nitinol to fully recover from strains in excess of $6 \%$ makes this material particularly attractive for percutaneous cardiovascular devices such as stent grafts, peripheral stents, and heart valves $[1,2]$. With widespread use of Nitinol in medical devices, it is important to understand the corrosion susceptibility of Nitinol, especially if corrosion results in adverse events such as loss of the implant's mechanical integrity and nickel (Ni) toxicity/ sensitization. The in vitro corrosion resistance of Nitinol has been shown to be highly dependent on surface processing $[3,4]$. For example, previous studies have shown that surface treatments such as electropolishing and passivation of stents increase the resistance to localized corrosion/pitting during potentiodynamic polarization experiments $[5,6]$. This increased in vitro pitting resistance has been attributed to the presence of an oxide layer that has a more desired chemistry, composition, and uniformity. In particular, titanium oxide layer thickness and exposed Ni-rich phases (i.e., regions characterized by an elevated $\mathrm{Ni}$ to Ti ratio, as compared to the base material) have been shown to be important factors in the corrosion behavior of Nitinol [7, 8]. Zhu et al. found a dramatic decrease in 
breakdown potentials when the oxide layer thickness ranged from 0.1 to 10 microns for electropolished Nitinol [8]. In addition, nickel particles within the oxide layer or $\mathrm{Ni}$ rich phases residing below the surface may be a source for Ni release over time. Previous immersion testing demonstrated that polished Nitinol exhibits similar $\mathrm{Ni}$ release behavior to stainless steels and cobalt-based alloys $[9,10]$. Longer immersion studies (up to 6 months) found that surface treatments such as polishing and/or passivation of Nitinol wires can reduce Ni release compared to untreated controls [11-14]. Wire immersion tests have shown that thicker oxides, surface $\mathrm{Ni}$ particles, and Ni-rich phases contribute to increased Ni dissolution $[15,16]$.

For percutaneous devices, the effects of constraining the device in a delivery system (i.e., radial compression) and then releasing the constraint during deployment may damage the oxide layer. Strains from radial compression have been reported up to $10 \%$ in endovascular devices and 6-8 \% for percutaneous heart valves [17, 18]. Although Nitinol may be able to sustain this large forward and reverse mechanical strain excursion, the non-superelastic oxide layer may crack under large strains, creating a conduit for exposure of the nickel-rich phases to the in vivo environment which will result in increased $\mathrm{Ni}$ release and decreased resistance to pitting. Zhu et al. found that $3 \%$ bending strains in wires with thick oxides caused cracking resulting in a substantial decrease in breakdown potentials [8]. However, Shabalovskaya et al. found that Nitinol wires subjected to $3 \%$ strain can maintain corrosion resistance [19]. Therefore, the consequences of stent radial compression on $\mathrm{Ni}$ release (uniform corrosion) for different surface compositions remain unclear, particularly for stents with thicker thermal oxide layers that possess subsurface Ni-rich phases.

Although previous studies demonstrate the importance of surface processing on the composition of the oxide layer and its impact on localized corrosion behavior of Nitinol, the comparative performance in pitting and uniform corrosion susceptibility between different surface compositions has yet to be investigated. This study aims to address the knowledge gap by elucidating the relationships between post-manufacturing surface composition, the effect of radial compression on in vitro corrosion performance, and in vitro tests for pitting and uniform corrosion.

\section{Methods}

\section{Stent Manufacturing and Characterization}

The stents used in this study were $8 \mathrm{~mm}$ diameter $\times 30 \mathrm{~mm}$ length (approximately $6.35 \mathrm{~cm}^{2}$ of total surface area) open source stents provided by Nitinol
Devices and Components (NDC). The laser-cut stents were divided into five groups, differing in material surface condition and processing steps: oxidized tube shape set in salt pot (OT), ground tube shape set in air furnace (AF), ground tube shape set in salt pot (SP), ground tube shape set in salt pot followed by mechanical polish (MP), and ground tube shape set in salt pot followed by electropolish (EP) (Fig. 1). With the exception of the OT group, laser cut samples were chemically polished in a proprietary solution after deburring, desludging, and honing, to remove the heat-affected zone from laser cutting. Following the chemical polishing step, stress relief and shape setting heat treatments for the OT, SP, MP, and EP groups were performed in a salt pot at $505{ }^{\circ} \mathrm{C}$. Heat treatments for the $\mathrm{AF}$ group were performed in a Thermodyne furnace in air, with a stress relief step at $540{ }^{\circ} \mathrm{C}$, expansion steps at $505{ }^{\circ} \mathrm{C}$, and an $A_{\mathrm{f}}$ tuning step at $550{ }^{\circ} \mathrm{C}$. Following the heat treatments, MP stents were ultrasonically cleaned in a mild detergent for $3 \mathrm{~min}$ (Micro90, International Products), chemically etched in a proprietary etching solution, chemically polished in a proprietary polishing solution, and burnished with 400 grit sandpaper. The EP stents were ultrasonically cleaned, chemically etched, and electropolished in a proprietary electropolishing solution. After heat treatment, the OT, AF, and SP stents were not polished, only ultrasonically cleaned in order to preserve the thermal surface oxide layer on the stents. A summary of the processing steps can be found in Table 1 .

Auger analysis of the stents was performed at Evans Analytical Group (Sunnyvale, CA) to characterize the oxide layer thickness and composition formed as a result of the various processing steps. A random spot on the outer diameter surface of one stent from each group was chosen for analysis. Oxide thickness was determined based on the full width at half maximum (FWHM) method.

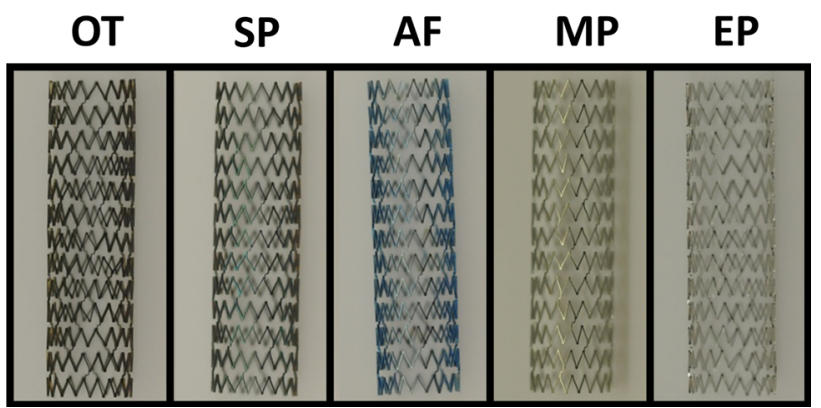

Fig. 1 Photographs of NDC open source stents. Stents were fabricated using the following surface treatments: oxidized tube (OT), air furnace (AF), salt pot (SP), mechanical polish (MP), and electropolish (EP) 
Table 1 Manufacturing processes for oxidized tube (OT), air furnace (AF), salt pot (SP), mechanical polish (MP), and electropolish (EP)

\begin{tabular}{llllll}
\hline & $\mathrm{OT}$ & $\mathrm{AF}$ & $\mathrm{SP}$ & $\mathrm{MP}$ & $\mathrm{EP}$ \\
\hline Stress relief & $505{ }^{\circ} \mathrm{C}$ & $540{ }^{\circ} \mathrm{C}$ & $505{ }^{\circ} \mathrm{C}$ & $505{ }^{\circ} \mathrm{C}$ & $505{ }^{\circ} \mathrm{C}$ \\
Expansion & $505{ }^{\circ} \mathrm{C}$ & $505^{\circ} \mathrm{C}$ & $505{ }^{\circ} \mathrm{C}$ & $505{ }^{\circ} \mathrm{C}$ & $505{ }^{\circ} \mathrm{C}$ \\
$A_{\mathrm{f}}$ tuning & $505{ }^{\circ} \mathrm{C}$ & $550{ }^{\circ} \mathrm{C}$ & $505{ }^{\circ} \mathrm{C}$ & $505{ }^{\circ} \mathrm{C}$ & $505{ }^{\circ} \mathrm{C}$ \\
Finishing & Ultrasonic clean & Ultrasonic clean & Ultrasonic clean & Etch burnish & Etch electropolish \\
\hline
\end{tabular}

\section{Pitting Corrosion Testing}

Cyclic potentiodynamic polarization testing per ASTM F2129-08 (Standard test method for conducting cyclic potentiodynamic polarization measurements to determine the corrosion susceptibility of small implant devices) was conducted on stents ( $n=6$ per surface treatment) to assess pitting corrosion potential in vitro. Phosphate buffered saline (PBS) (BP661-50, Fisher Scientific, Pittsburgh, PA) was used as the electrolyte solution at a temperature of $37 \pm 2{ }^{\circ} \mathrm{C}$. PBS was deaerated using nitrogen gas at $150 \mathrm{~mL} / \mathrm{min}$. After stents were immersed in solution for $1 \mathrm{~h}$ to obtain the rest potential, the potentiodynamic scan was initiated in the positive direction at a scan rate of $1 \mathrm{mV} / \mathrm{s}$. The scan was reversed at either the vertex potential $(1000 \mathrm{mV})$ or when the current density became two decades higher than the current density at the breakdown potential. The rest potential (Er) and breakdown potential $(\mathrm{Eb})$ were recorded for each stent.

\section{Immersion Testing}

All containers and instruments used for the testing, handling, and storage of specimens were acid washed using a $10 \% \mathrm{HNO}_{3}$ solution (A509-P212, Fisher Scientific, Pittsburgh, PA) prior to use. Immersion testing was conducted on a total of fifty stents. To understand the effects of radial compression on nickel release, stents from each group ( $n=5$ per surface condition) were radially compressed once to an outer diameter of $2 \mathrm{~mm}$ (corresponding to a $7 \%$ maximum local strain from finite element analysis) prior to immersion (RMC Radial Compression Station, Blockwise Engineering LLC, Tempe, AZ) and compared to a group of stents that were not radially compressed $(n=5$ per surface treatment). Each stent was placed in a $60 \mathrm{~mL}$ polypropylene container filled with $30 \mathrm{~mL}$ of PBS (pH $7.4 \pm 0.1$ ), resulting in a stent surface area to PBS volume ratio of $0.21 \mathrm{~cm}^{2} / \mathrm{mL}$. All containers were placed into an environmental chamber (Hotpack, Philadelphia, PA) at $37{ }^{\circ} \mathrm{C}$ for a total of 60 days. PBS samples for nickel ion measurements were taken at ten time points: Days 1, 2, 3, 5, 7, 14, 21, 30, 45 , and 60 . At each time point, the stent was removed from its test container and immediately placed into a new container of fresh PBS. A $2 \mathrm{~mL}$ aliquot was taken using a trace metal certified pipette tip (MLA Pipette Tips, VistaLabs
Technologies, Brewster, NY) for each sample. A $4 \%$ $\mathrm{HNO}_{3}$ solution (Optima A467-P500, Fisher Scientific, Pittsburgh, PA) was added to all completed PBS samples. Nitric acid addition ensured that any adsorbed nickel would be dissolved back into solution. All samples were stored in an area known to be free from trace metal contamination until $\mathrm{Ni}$ ion analyses were performed. PBS controls (blank) were used throughout the duration of immersion to monitor for contamination issues. After immersion testing, all stents were removed from PBS and transferred into corresponding polypropylene tubes filled with $30 \mathrm{~mL}$ of ultra-pure DI water. Tubes were gently agitated on an orbital shaker at room temperature for $1 \mathrm{~h}$. After rinsing, each device was removed from its tube and allowed to air dry before visual analysis.

\section{Ni Ion Quantification}

A Thermo X-Series II quadruple inductively coupled plasma mass spectrometer (ICP-MS) was used to determine the total concentration of nickel in the stock solutions. ICPMS measurements were conducted in collision cell technology (CCT) mode with a gas of $\mathrm{He} / \mathrm{H}_{2}(99.999 \%$ purity, v/v: $93 \% / 7 \%)$. The machine was tuned with $1 \mathrm{ppb}$ Tune A solution (Thermo Fisher) to meet the required performance. NIST 3136 Nickel Standard Solution was used as a calibration standard. The limit of detection for nickel was calculated to be $0.1 \mathrm{ppb}$. A $50 \mathrm{ppb}$ internal standard solution (VHG, contains $\mathrm{Bi}, \mathrm{Ga}, \mathrm{In}, \mathrm{Sc}, \mathrm{Tb}$, and $\mathrm{Y}$ ) was introduced along with the respective samples through a T-connector to correct signal drift and matrix effects. A $2 \% \mathrm{HNO}_{3}$ was used as a diluent for all solutions and was used as a rinse solution. All nickel samples were prepared in the range of $0-100 \mathrm{ppb}$. If any exceeded this range, additional dilution with $2 \% \mathrm{HNO}_{3}$ was performed. An additional calibration standard was run as an unknown during the experiment for quality control purposes. $\mathrm{Ni}$ spike and recovery testing were performed to ensure $\mathrm{Ni}$ quantification with the procedure was robust. Four PBS solutions were prepared using the following procedure: NIST $3136 \mathrm{Ni}$ standard solution $(10 \mathrm{mg} / \mathrm{mL})$ was first diluted with $2 \% \mathrm{HNO}_{3}$ to $1 \mathrm{ppm}$ and then further diluted with PBS to $100 \mathrm{ppb}$. Two containers of PBS spiked with $100 \mathrm{ppb}$ were measured with ICP-MS immediately. The other two bottles were measured after 15 days of 
incubation at $37{ }^{\circ} \mathrm{C}$. The Ni recovery for both immediate and incubated solutions ranged from 99 to $102 \mathrm{ppb}$. In addition, incubated PBS blanks used throughout the immersion testing as internal controls consistently possessed $\mathrm{Ni}$ values below $0.5 \mathrm{ppb}$. These results demonstrated that the procedure used for immersion testing was adequate for evaluating nickel ion release of Nitinol stents with varying surface compositions.

\section{Visual Inspection}

Prior to immersion, one device from each surface treatment group (AF, EP, MP, OT, SP) for both radially compressed and non-compressed stents was visually inspected using scanning electron microscopy (SEM) (JSM-6390LV, JOEL USA, Inc., Peabody, MA). Post-immersion, the two stents with highest $\mathrm{Ni}$ release concentrations from each group were imaged for corrosion at a minimum of $300 \times$ magnification.

\section{Statistics}

One-way ANOVA with $T$ tests was used to assess differences between surface treatment groups and radial compression effects. All data are presented as mean \pm SD. $p$ values $<0.05$ were considered significant. In addition, linear log-weighted regression analyses were performed to correlate cumulative nickel release to oxide layer thickness.

\section{Results}

\section{Stent Characterization and Pitting Corrosion Testing}

Each group of processed stents possessed unique surface composition and chemistry (Fig. 2), and pitting corrosion resistance (Table 2). The OT group had an oxide layer that exceeded the sputter depth of the Auger analysis $(>3500 \mathrm{~nm})$ and possessed the lowest resistance to pitting corrosion $(\mathrm{Eb}=-117 \pm 15 \mathrm{mV})$ during ASTM F2129 testing. Due to the oxide layer thickness, a nickel-rich sublayer could not be detected in the OT sample, but a concentration of nickel was present on the surface. The AF oxide layer was measured to be approximately $130 \mathrm{~nm}$, with the slight presence of a nickel-rich layer and a pitting corrosion resistance of $\mathrm{Eb}=144 \pm 73 \mathrm{mV}$. The SP group had a thick oxide layer $(420 \mathrm{~nm})$ and a high pitting corrosion resistance (i.e., no breakdown up to the vertex potential of $1000 \mathrm{mV}$ ), despite the presence of a nickel-rich layer beneath the titanium oxide. Both the MP and the EP had thin oxide layers ( 4.3 and $3.8 \mathrm{~nm}$, respectively) with no presence of a nickel-rich layer. The MP group possessed high pitting corrosion resistance $(\mathrm{Eb}=832 \pm 256 \mathrm{mV})$, while the EP stents did not exhibit breakdown up to $1000 \mathrm{mV}$.

\section{Nickel Release}

OT stents, regardless of whether they were subjected to compression (designated as "C" in Fig. 3) or not compressed ("NC" designation in Fig. 3), exhibited the greatest amount of $\mathrm{Ni}$ release throughout 60 days of immersion with cumulative $\mathrm{Ni}$ release values that were $5-10 \times$ higher than those measured for any other group $(p<0.001)$. In addition, within each set $(\mathrm{C}$ or NC), SP and AF stents released the second and third most Ni, respectively. The average AF Ni release was lower $(p<0.006)$ than SP stents after day 2 within each set (C and NC). Ni release for all MP and EP stents was consistently low, and cumulative release fell below $1100 \mathrm{ng}$ at the conclusion of the immersion period. Within the $\mathrm{NC}$ group, the average $\mathrm{Ni}$ release measured for AF-NC stents was not significantly different $(p>0.207)$ than that measured for the EP-NC group. In contrast, $\mathrm{AF}-\mathrm{C}$ stents exhibited significantly higher Ni release $(p<0.001)$ as compared to MP-C and EP-C groups.

Groups with the thickest oxide layers (OT and SP) possessed a significantly greater $(p \leq 0.001)$ cumulative $\mathrm{Ni}$ release for radially compressed stents compared to noncompressed stents at every time point (Fig. 3). This trend was reversed for all other groups (AF, MP, EP) which resulted in a greater cumulative Ni release for non-compressed stents compared to compressed stents (Fig. 3). At each time point, cumulative $\mathrm{Ni}$ release was higher $(p \leq 0.011)$ for stents which were not radially compressed as compared to those which were compressed in the AF, MP and EP groups with two exceptions. On Day 14, while the average Ni release for MP-NC stents was greater than MP-C stents, this did not reach the level of statistical significance $(p>0.507)$. In addition, the average $\mathrm{Ni}$ release for AF-NC stents for the first 3 time points (Day 1, 2, and 3) was greater than that of AF-C but also did not reach the level of statistical significance $(p>0.065)$.

\section{Visual Analysis}

Visual inspection using optical microscopy and SEM revealed no signs of pitting corrosion or significant uniform corrosion (i.e., measureable mass loss) post 60-day immersion testing for all groups when compared to preimmersion samples. The surface appearance and morphology were similar on pre-immersion and post-tested samples for all groups (Fig. 4). 


\section{OT}

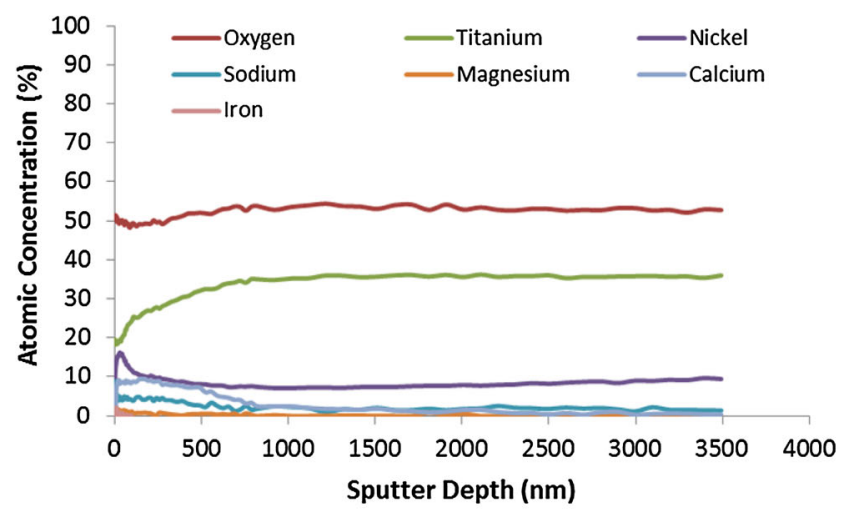

SP

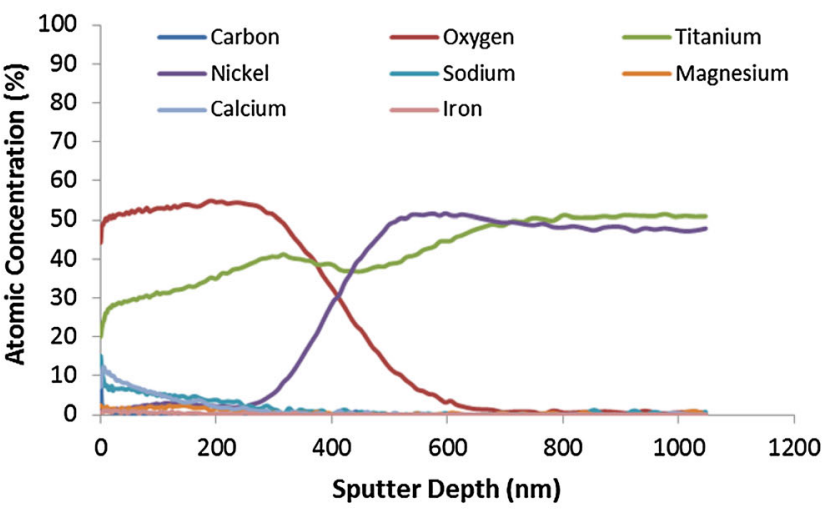

$\mathrm{AF}$

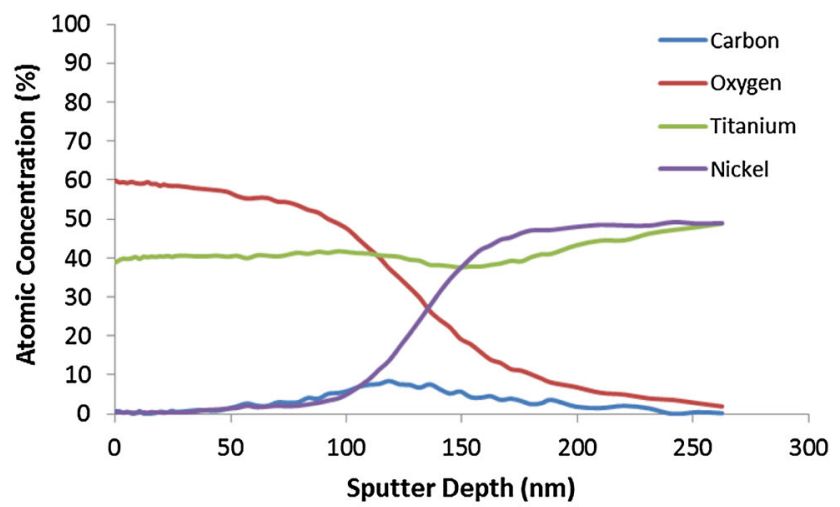

MP

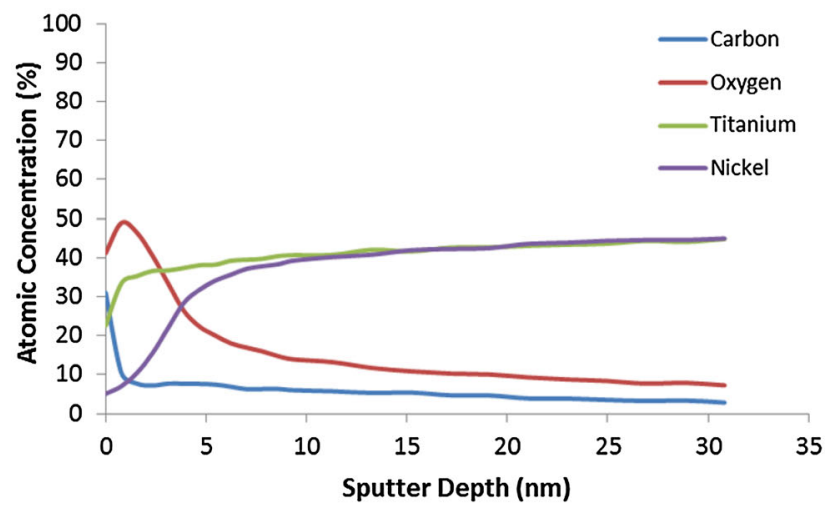

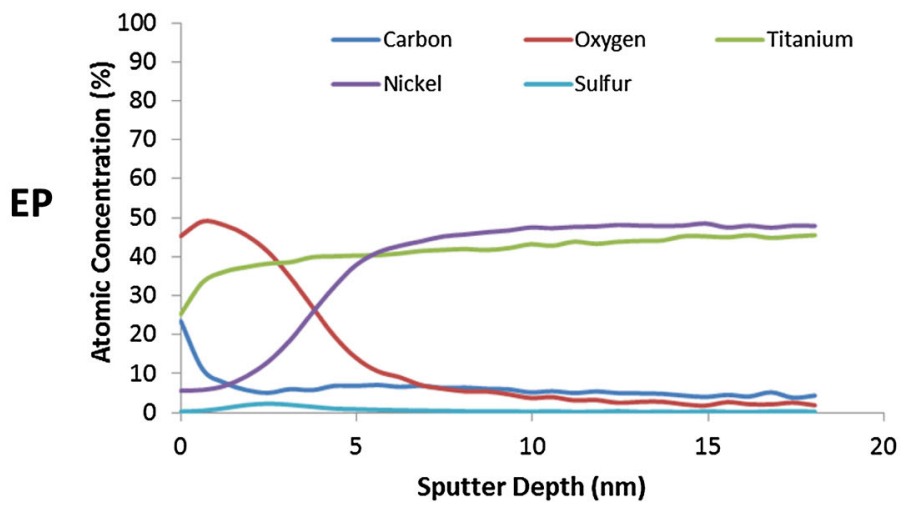

Fig. 2 Auger depth plots used to determine oxide layer chemistry and thickness: OT > $3500 \mathrm{~nm}$; AF $130 \mathrm{~nm}$; SP $420 \mathrm{~nm}$; MP $4.3 \mathrm{~nm}$; EP $3.8 \mathrm{~nm}$

Table 2 Rest (Er) and breakdown (Eb) potentials for potentiodynamic polarization corrosion testing

\begin{tabular}{llllll}
\hline & OT & AF & SP & MP & EP \\
\hline$E_{\mathrm{r}(\mathrm{mV})}$ & $-504.8 \pm 6.0$ & $-160.1 \pm 42.5$ & $-120.7 \pm 87.0$ & $-74.2 \pm 28.6$ & $-76.0 \pm 61.6 \mathrm{mV}$ \\
$E_{\mathrm{b}}$ & $-117.0 \pm 14.5 \mathrm{mV}$ & $143.6 \pm 73.0 \mathrm{mV}$ & $\mathrm{NB}$ & $831.7 \pm 256.0 \mathrm{mV}$ & $\mathrm{NB}$ \\
\hline
\end{tabular}

NB indicates no breakdown was observed up to vertex potential. Values reported are mean \pm standard deviation 


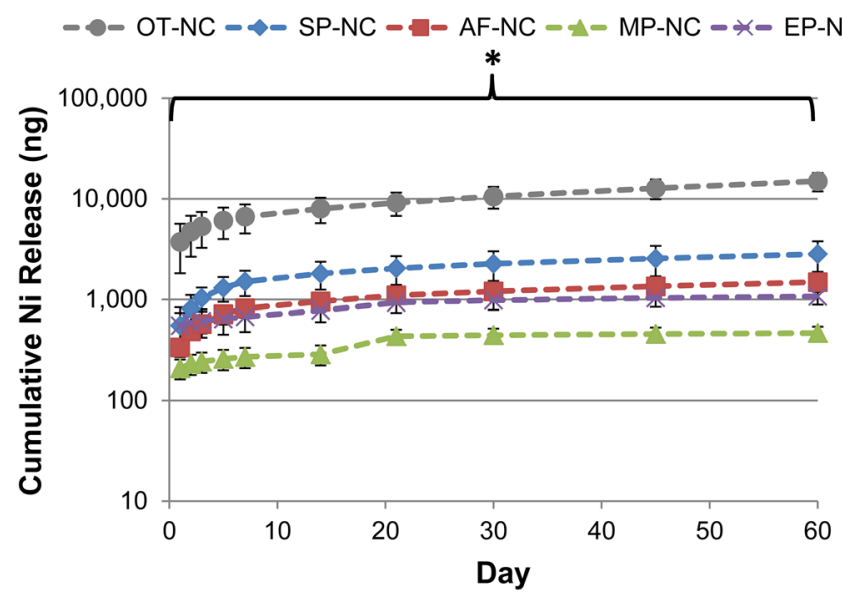

Fig. 3 Cumulative Ni released (ng) per stent over a 60-day period for the (left) Not Compressed ("-NC") stent set and (right) Compressed (“-C") stent sets. In the NC set, OT and SP groups are significantly

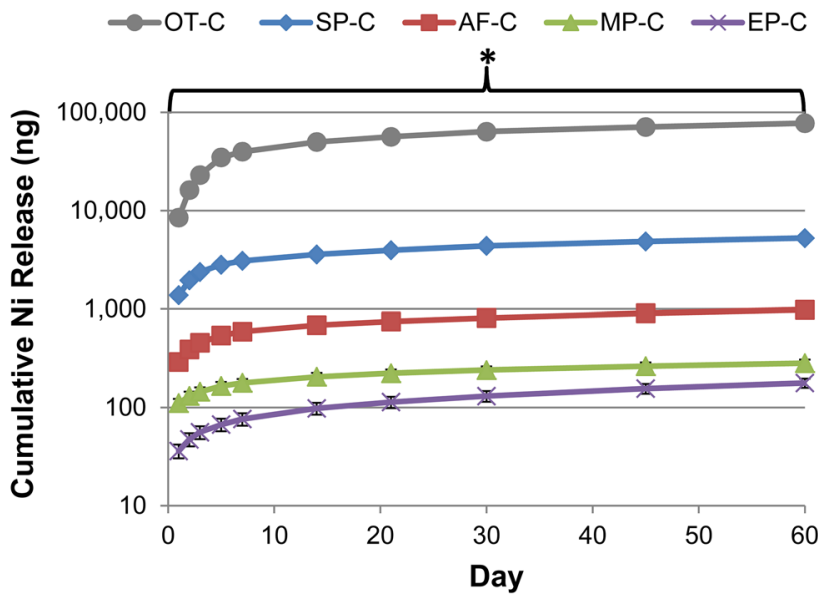

different $(* p<0.001)$ than lower groups. In the C set, OT, SP, and $\mathrm{AF}$ groups are significantly different $(* p<0.001)$ than lower groups
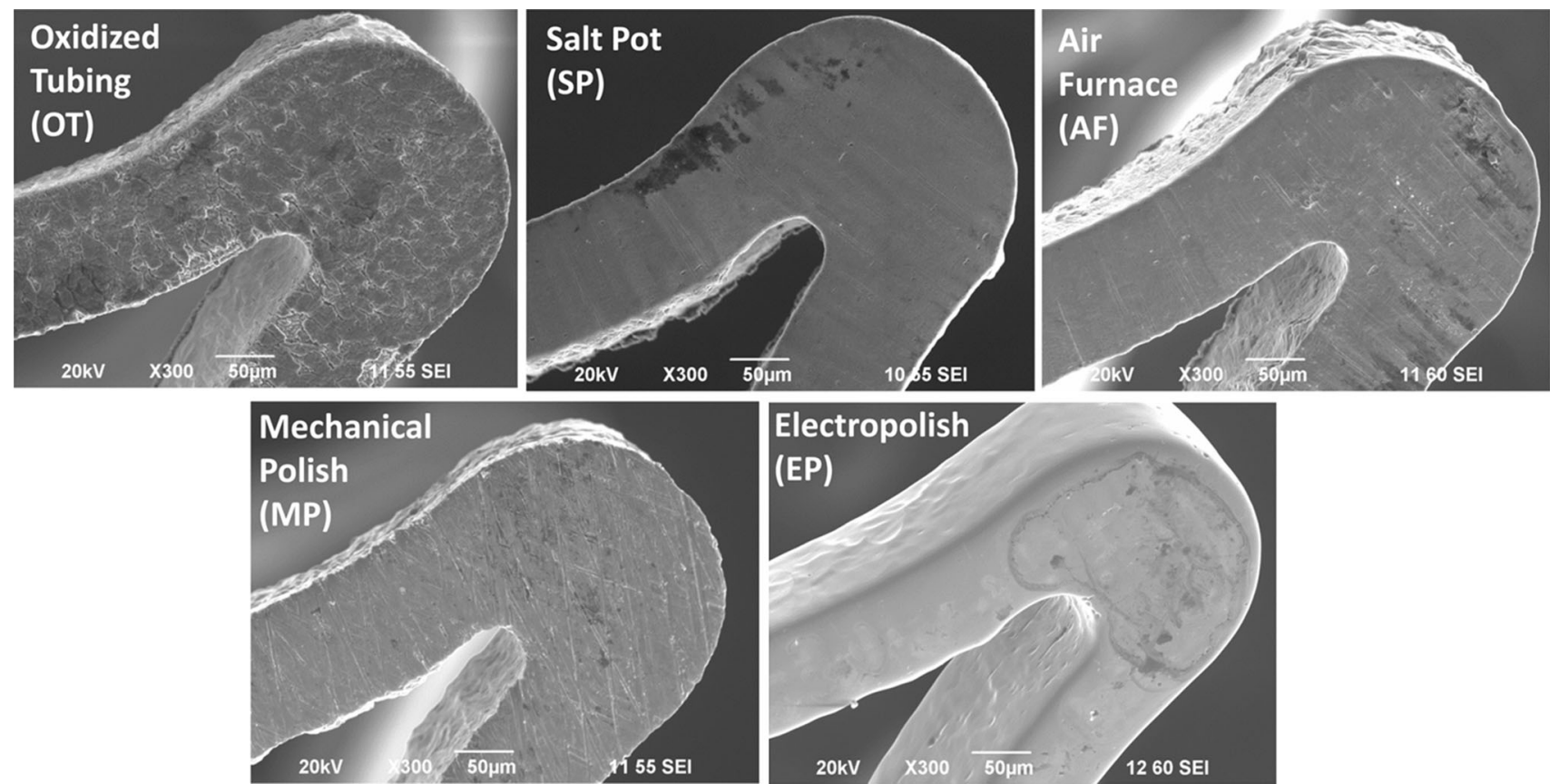

Fig. 4 Representative SEM image ( $\times 300)$ of each stent group: oxidized tube $(\mathrm{OT})$, air furnace $(\mathrm{AF})$, salt pot $(\mathrm{SP})$, mechanical polish $(\mathrm{MP})$, and electropolish (EP)

\section{Discussion}

It is well accepted that surface processing of Nitinol impacts in vitro pitting resistance and nickel release. However, these correlations were obtained mainly from studies using wires and may not be directly translatable to cardiovascular stents that are typically laser cut from tubes and radially compressed prior to implantation. In our study, we manufactured laser-cut stents with both standard and non-standard surface treatments to directly correlate with in vitro localized and uniform corrosion. Our results demonstrated that there is a significant impact of surface processing on both pitting corrosion and $\mathrm{Ni}$ leaching. As expected, removal of the thermal oxide and nickel-rich phase using polishing of stents resulted in higher pitting corrosion resistance and lower $\mathrm{Ni}$ release compared to those without polishing. Non-polished SP stents exhibited high pitting corrosion resistance, but had the second highest Ni levels during immersion testing. We suspect that the thick protective oxide layer likely prevented pitting 
during polarization testing, but the subsurface Ni-rich regions appear to have allowed for greater $\mathrm{Ni}$ release as compared to the polished stents. Radial compression of the $\mathrm{SP}$ and the OT stents further increased Ni release compared to non-compressed stents. SEM images qualitatively verified more cracking of the oxide layer at the intrados of radially compressed SP stents compared to the non-compressed group (Fig. 5). It is important to note, however, that radially compressing stents did not always release a greater amount of Ni. Interestingly, we found that MP, EP, and AF stents had comparable or lower Ni release in the radially compressed stents when compared to their noncompressed counterpart. For the polished groups, crackinginduced increase in nickel release would not be expected due to a thin oxide layer and lack of a subsurface nickelrich region. However, the AF stent results were an unexpected finding. One explanation may be the inherent variability in using an AF during heat treatment. Temperatures within the furnace are less controlled than in a salt pot and the process is more susceptible to operator inconsistency. This may have resulted in larger differences in the oxide layer composition and chemistry between stents in the $\mathrm{AF}$ group, as evidenced by the slightly defined nickel-rich layer in the AF stent depth profile as compared to the welldefined nickel-rich layer observed in the SP stent depth profile. In fact, we observed slight variations in color between the radially compressed and non-compressed stents, which supports the notion that the oxide layer thickness and composition may vary for AF stents.

Overall these results suggest that additional $\mathrm{Ni}$ release due to radial compression is highly dependent on surface processing and that thicker oxide layers may be susceptible to cracking and exposing nickel-rich regions when compressed. Moreover, within each set (radially compressed and not compressed), Ni release was strongly correlated $\left(R^{2}>0.94\right)$ to oxide layer thickness (Fig. 6). The positive correlation between cumulative $\mathrm{Ni}$ release and the oxide layer thickness suggests there may be a transition between

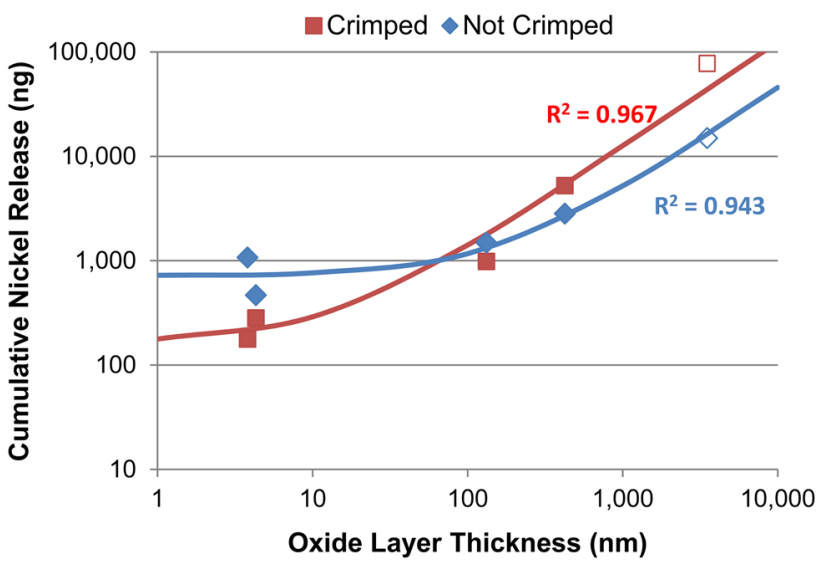

Fig. 6 Correlation of total cumulative Ni release and oxide layer thickness for not compressed and compressed stent groups. Linear log-weighted regressions resulted in good correlation $\left(R^{2}>0.94\right)$ for non-compressed (Ni release $=4.5 \times$ oxide thickness +720.3$)$ and compressed $(\mathrm{Ni}$ release $=12.5 \times$ oxide thickness +165$)$ stents. With OT stent data removed (open square and triangle data points), correlations $\left(R^{2}>0.79\right)$ changed for non-compressed $(\mathrm{Ni}$ release $=5.3 \times$ oxide thickness +696.1$)$ and compressed $(\mathrm{Ni}$ release $=8.9 \times$ oxide thickness +183.4 ) stents

"thick" and "thin" oxide layer where radial compression may increase Ni release. Even if the OT group is removed from the regression analysis (since oxide layer thickness was not precisely known), moderate correlations $\left(R^{2}>0.79\right)$ still exist within the remaining data points. In order to fully characterize the relationship between oxide thickness, Ni release, and mechanical strain, further surface characterization, particularly for the AF group, needs to be performed.

Corrosion (both localized and uniform) in implantable devices is an important consideration during safety assessments of medical devices, particularly the need to maintain mechanical integrity of the device and ensure biocompatibility for the patient. Mitigating toxicity concerns is especially important for $\mathrm{Ni}$ released in the body. There have been many studies investigating the non-cancer
Fig. 5 SEM images $(\times 4000)$ of micro-cracks at the intrados of not compressed (left) and radially compressed (right) SP stents. Micro-cracks typical of non-compressed stents appear more prominent after radial compression (arrows)
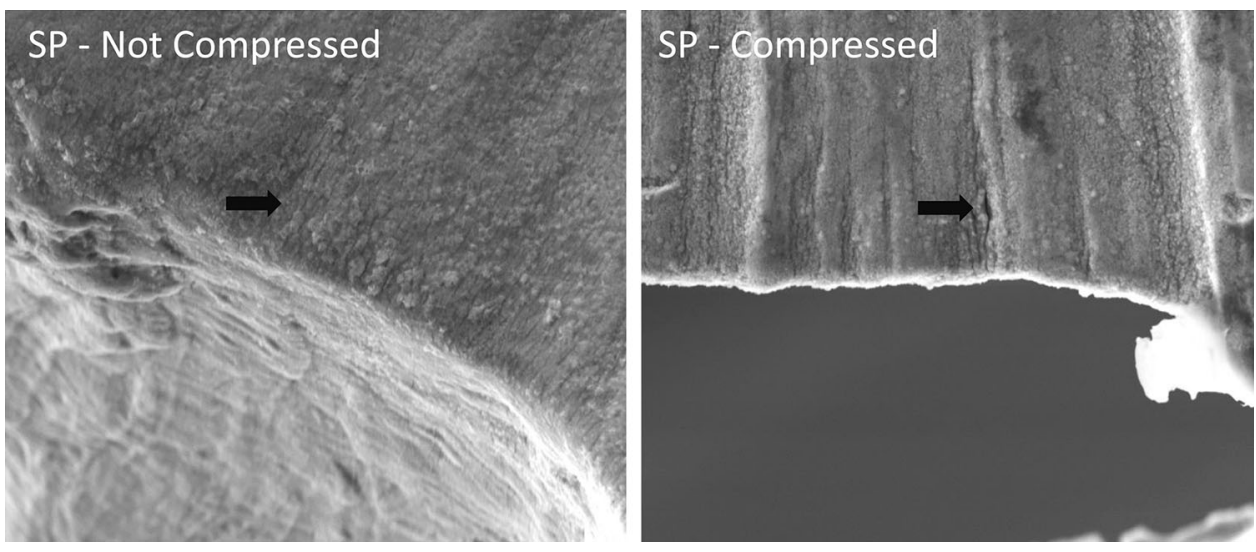
toxicity of nickel through various routes of exposure [2023]. However, for implantable nickel-based devices, there have been no definitive tolerable intake values reported. The US Pharmacopeia (USP) suggested a permissible daily exposure (PDE) for nickel as a metallic impurity in drug products to be $0.5 \mu \mathrm{g} / \mathrm{kg} / \mathrm{day}$. For a $70 \mathrm{~kg}$ person, the PDE would be $35 \mu \mathrm{g} /$ day, which is greater than the highest $\mathrm{Ni}$ release $(9 \mu \mathrm{g} / \mathrm{day})$ for stents used in this study. Although this value is below the USP's PDE, testing of multiple stents such as those in an overlapped condition or larger sized stents such as those implanted into the superficial femoral artery may generate higher nickel release rates than those reported in this study.

A few limitations must be considered when interpreting the findings of this study. First, radial compression was performed only at one level. Although the radial compression used in this study is in the range of levels used clinically, additional research into the impact of different compression levels on Ni release is needed. In addition, the Ni release reported for this study may not be indicative of the in vivo environment as these stents were immersed in PBS and left in an unconstrained, static condition. Although PBS does not fully simulate blood and therefore may not be indicative of $\mathrm{Ni}$ values seen in vivo, this test method provides a repeatable basis for relative comparisons between groups. Finally, it is unknown what clinical sequelae, if any, would arise from the levels of nickel reported in this study. An animal study is currently underway to investigate the biological consequences of $\mathrm{Ni}$ release systemically as well as locally on arterial vessels.

In summary, this study demonstrates that surface processing affects both uniform and pitting corrosion resistance for laser-cut Nitinol stents, regardless of whether or not they are radially compressed. Devices with polishing as a final surface treatment (i.e., the MP and EP groups) were characterized by thin oxide layers, exhibited high corrosion resistance (localized and uniform), and were not negatively affected by the radial compression process. Conversely, OT stents had minimal surface processing resulting in a very thick, complex oxide, low pitting, and uniform corrosion resistance, and were negatively affected by radial compression. For AF and SP processed stents, correlations between uniform and pitting corrosion resistance and the impact of radial compression were not as straightforward and may be attributed to interplay of several factors such as oxide layer thickness, nickel-rich regions, and oxide uniformity. Overall, this study illustrates the importance of surface treatment steps such as polishing on a device's corrosion resistance and response to radial compression.

Acknowledgments This study was funded by the FDA's Critical Path Initiative. The authors would like to thank FDA researchers Matthew Di Prima for pitting corrosion assistance, John Bouck for immersion testing method development, and David Saylor for manuscript review. The authors would like to acknowledge the FDA White Oak Nanotechnology Core Facility for instrument use, scientific and technical assistance. We also acknowledge NDC's Karen Tan, Anna Johnson, and Navjeet Gill for assistance with stent processing and corrosion testing.

\section{References}

1. Shaw JA, Kyriakides S (1995) Thermomechanical aspects of NiTi. J Mech Phys Solids 43:1243-1281

2. Duerig TM, Melton KN, Stockel D, Wayman CM (1990) Engineering aspects of shape memory alloys. Butterworth-Heinemann, London

3. Shabalovskaya SA (2002) Surface, corrosion and biocompatibility aspects of Nitinol as an implant material. Biomed Mater Eng 12:69-109

4. Shabalovskaya S, Rondelli G, Anderegg J, Xiong JP, Wu M (2004) Comparative corrosion performance of black oxide, sandblasted, and fine-drawn nitinol wires in potentiodynamic and potentiostatic tests: effects of chemical etching and electropolishing. J Biomed Mater Res B Appl Biomater 69:223-231

5. Trepanier C, Tabrizian M, Yahia LH, Bilodeau L, Piron DL (1998) Effect of modification of oxide layer on NiTi stent corrosion resistance. J Biomed Mater Res 43:433-440

6. O'Brien B, Carroll W, Kelly M (2002) Passivation of nitinol wire for vascular implants: a demonstration of the benefits. Biomaterials 23:1739-1748

7. Shabalovskaya S, Anderegg J, Van Humbeeck J (2008) Critical overview of Nitinol surfaces and their modifications for medical applications. Acta Biomater 4:447-467

8. Zhu L, Trepanier C, Pelton A, Fino JM (2003) Oxidation of nitinol and its effect on corrosion resistance. In: ASM Materials and Processes for Medical Devices, pp. 156-161

9. Thierry B, Tabrizian M, Trepanier C, Savadogo O, Yahia LH (2000) Effect of surface treatment and sterilization processes on the corrosion behavior of NiTi shape memory alloy. J Biomed Mater Res 51:685-693

10. Okazaki Y, Gotoh E (2008) Metal release from stainless steel, $\mathrm{Co}-\mathrm{Cr}-\mathrm{Mo}-\mathrm{Ni}-\mathrm{Fe}$ and $\mathrm{Ni}-\mathrm{Ti}$ alloys in vascular implants. Corros Sci 50:3429-3438

11. Wu S, Chu PK, Liu X, Chung C, Ho J, Chu C, Tjong S, Yeung K, Lu W, Cheung K (2006) Surface characteristics, mechanical properties, and cytocompatibility of oxygen plasma-implanted porous nickel titanium shape memory alloy. J Biomed Mater Res Part A 79:139-146

12. Pérez LM, Gracia-Villa L, Puértolas JA, Arruebo M, Irusta S, Santamaria J (2009) Effect of Nitinol surface treatments on its physico-chemical properties. J Biomed Mater Res B Appl Biomater 91:337-347

13. Cui Z, Man H, Yang X (2005) The corrosion and nickel release behavior of laser surface-melted NiTi shape memory alloy in Hanks' solution. Surf Coat Technol 192:347-353

14. Wever DJ, Veldhuizen AG, de Vries J, Busscher HJ, Uges DRA, van Horn JR (1998) Electrochemical and surface characterization of a nickel-titanium alloy. Biomaterials 19:761-769

15. Clarke B, Carroll W, Rochev Y, Hynes M, Bradley D, Plumley D (2006) Influence of nitinol wire surface treatment on oxide thickness and composition and its subsequent effect on corrosion resistance and nickel ion release. J Biomed Mater Res Part A 79:61-70

16. Shabalovskaya SA, Tian H, Anderegg JW, Schryvers DU, Carroll WU, Van Humbeeck J (2009) The influence of surface oxides on 
the distribution and release of nickel from Nitinol wires. Biomaterials 30:468-477

17. Kleinstreuer C, Li Z, Basciano C, Seelecke S, Farber M (2008) Computational mechanics of Nitinol stent grafts. J Biomech 41:2370-2378

18. Kumar GP, Mathew L (2012) Self-expanding aortic valve stent: material optimization. Comput Biol Med 42:1060-1063

19. Shabalovskaya SA, Rondelli GC, Undisz AL, Anderegg JW, Burleigh TD, Rettenmayr ME (2009) The electrochemical characteristics of native Nitinol surfaces. Biomaterials 30:3662-3671

20. Pereira M, Pereira M, Sousa J (1998) Evaluation of nickel toxicity on liver, spleen, and kidney of mice after administration of high-dose metal ion. J Biomed Mater Res 40:40-47
21. Smith MK, George EL, Stober JA, Feng H, Kimmel G (1993) Perinatal toxicity associated with nickel chloride exposure. Environ Res 61:200-211

22. S-j W, D-m P, B-s C (2002) Variation of systolic blood pressure in rats exposed to cadmium and nickel. Environ Res 88:116-119

23. Vyskočil A, Senft V, Viau C, Cížková M, Kohout J (1994) Biochemical renal changes in workers exposed to soluble nickel compounds. Hum Exp Toxicol 13:257-261 